\title{
Elektrochemische Aktivitätsbestimmung der humanen Monoaminooxidase B durch selektive Bindung
}

\author{
S. Höfs ${ }^{1}$, T. Greiner ${ }^{1}$, G. Göbel' ${ }^{1}$, A. Talke ${ }^{2}$, U. Ahnert ${ }^{2}$, F. Lisdat ${ }^{1}$ \\ ${ }^{1}$ Biosystems Technology, Technical University Wildau, WIIdau, Germany \\ ${ }^{2}$ Biotez GmbH, Berlin, Germany \\ Kontakt: ggoebel@th-wildau.de
}

\section{Einleitung}

\begin{abstract}
Morbus Parkinson gehört zu den weltweit häufigsten neurodegenerativen Erkrankungen [1]. Der Verlust von Neuronen in der Substantia nigra führt zu einem Dopaminmangel im Gehirn [2], der die Signalleitung beeinträchtigt und damit die Parkinson-typischen Bewegungsstörungen verursacht. Kommt als weiteres Merkmal die intrazelluläre Akkumulation von $\alpha-S y n u c l e i n ~ h i n z u$, gilt die Diagnose von Parkinson als gesichert [3].

Derzeit gibt es keine Therapie, die Morbus Parkinson verhindern bzw. deren Fortschreiten aufhalten kann. Um die Lebensqualität der betroffenen Patienten zu verbessern, werden die Symptome durch das Einstellen des Dopaminspiegels behandelt. Die Gabe der Dopaminvorstufe L-Dopa in Verbindung mit Inhibitoren Dopamin-abbauender Enzyme führt im frühen Stadium der Erkrankung zu einer deutlichen Besserung [4]. Infolge der fortschreitenden Degeneration der dopaminergen Zellen verstärken sich allerdings im Laufe Zeit die motorischen Störungen wieder. Um die Dosierung der Medikamente zu optimieren, ist eine Messung der Aktivität der betreffenden Enzyme wünschenswert.
\end{abstract}

Diese Studie konzentriert sich auf die Aktivitätsbestimmung der Monoaminooxidase B (Mao B), die die Desaminierung von primären und sekundären aromatischen Aminen katalysiert [5].

In der Literatur sind verschiedene Methoden zur Aktivitätsbestimmung der Mao B beschrieben. In komplexen Medien wird häufig die Markierung mit C-14 eingesetzt [6], deren Anwendung jedoch besondere Vorsichtsmaßnahmen erfordert. Fluorometrische Assays detektieren das enzymatisch gebildete Wasserstoffperoxid. In komplexen biologischen Proben ist ihre Selektivität jedoch eingeschränkt.

Die elektrochemische Quantifizierung der Mao B-Aktivität ist Gegenstand nur weniger Publikationen. Reyes-Parada bestimmt die Aktivität der Mao B mittels HPLC und anschließender amperometrischer Detektion des aus 4-Dimethylaminophenethylamin gebildeten Reaktionsproduktes 4Dimethylaminophenylessigsäure [7].

Des Weiteren kann die Aktivitätsbestimmung der Mao B durch die selektive Detektion von Edukt oder Produkt an
PEDOT:NAFION modifizierten Kohlefaser-Elektroden erfolgen $^{8}$. Obwohl die Detektion von Catecholaminen in der Literatur intensiv bearbeitet wird, wird der selektiven Detektion dieser Botenstoffe in Gegenwart ihrer Metabolite allerdings wenig Beachtung geschenkt. So erlaubt fluor-dotiertes Zinnoxid die selektive Detektion von Dopamin nach der Umsetzung zu Methoxytyramin durch die Catechol-O-Methyltransferase [8].

Schließlich erlaubt auch das bei der enzymatischen Substratumsetzung entstehende Wasserstoffperoxid eine Quantifizierung der Aktivität. Hierfür eignen sich insbesondere auf Preußisch Blau-basierende Elektroden, die bereits bei geringen Überpotentialen die Reduktion des Wasserstoffperoxids erlauben [9]. Die polykristalline Struktur des Preußisch Blau ermöglicht kleinen Molekülen wie Wasserstoffperoxid das selektive Eindringen in die Gitterstruktur [10]. Diese vorteilhafte Eigenschaft soll hier mit der Fähigkeit von spezifischen Antikörpern zur selektiven Bindung der Mao B kombiniert werden.

\section{Methoden und Materialien}

\section{Wasserstoffperoxid-Messungen}

Die elektrochemischen Messungen erfolgten an dem Potentiostaten $\mathrm{CHI} 800$.

Vor der Kalibrierung der $\mathrm{H}_{2} \mathrm{O}_{2}$ Elektroden (Rusens, Russland), erfolgte eine elektrochemische Vorbehandlung mit $100 \mu \mathrm{M} \mathrm{H} \mathrm{H}_{2}$ in $100 \mathrm{mM}$ Phosphatpuffer $(\mathrm{pH} 7,2)$ mit $100 \mathrm{mM} \mathrm{KCl}$ und $1 \mathrm{mM}$ Benzylamin. Dafür wurden die Elektroden in einer Wall-Jet-Durchflusszelle von Rusens platziert, die in ein Fließsystem integriert war. Eine Peristaltikpumpe sorgte für eine Flussrate von $310 \mu \mathrm{l} / \mathrm{min}$. Zum Austausch der Lösungen wurde die Pumpe abgeschaltet, um Luftblasen im Fließsystem zu vermeiden.

Zunächst wurde ein Potential von $-100 \mathrm{mV}$ vs. $\mathrm{Ag} / \mathrm{AgCl}$ angelegt, welches die Reduktion des $\mathrm{H}_{2} \mathrm{O}_{2}$ erlaubt. Anschließend wurde der Puffer (100 mM Phosphat (pH 7,2), $100 \mathrm{mM} \mathrm{KCl}$ und $1 \mathrm{mM}$ Benzylamin) durch das Fließsystem gepumpt bis sich der Basisstrom stabilisiert hatte. Danach wurden für 5 min $100 \mu \mathrm{M} \mathrm{H}_{2} \mathrm{O}_{2}$ durch die Zelle geleitet und 
schließlich wurde das Fließsystem mit Puffer gespült, bis sich ein stabiler Basisstrom einstellte.

Im Anschluss erfolgte die Kalibrierung der Preußisch Blau-Elektroden in einem $\mathrm{H}_{2} \mathrm{O}_{2}$-Konzentrationsbereich von $0,5 \mu \mathrm{M}$ bis $20 \mu \mathrm{M}$ in dem oben angegebenen Puffer. Nach jeder Messung wurde das System gespült, bis wieder ein konstanter Basisstrom vorlag.

Da Sensitivität der Preußisch Blau-Elektroden variierte, wurde die Kalibrierung für neue jede Elektrode durchgeführt.

Vor den Mao B-Aktivitätsmessungen, wurden das Mao B Substrat Benzylamin sowie die Produkte der enzymatischen Reaktion $\mathrm{NH}_{3}$ und Benzaldehyd hinsichtlich ihres Einflusses auf das Messsignal untersucht. Dafür wurden $500 \mu \mathrm{l}$ der drei Verbindungen mit Konzentrationen im Bereich von $1 \mathrm{mM}$ bis $15 \mathrm{mM}$ mit $310 \mu \mathrm{l} / \mathrm{min}$ bei $-100 \mathrm{mV}$ vs $\mathrm{Ag} / \mathrm{AgCl}$ durch die Fließzelle geleitet.

Schließlich erfolgte die Aktivitätsbestimmung durch das Durchleiten von Substrat-Lösungen $(500 \mu \mathrm{l})$, die unterschiedlich lange in Kontakt mit der Mao B waren.

\section{Mao B-Anreicherung}

Um die Mao-B aus einer biologischen Probe zu isolieren wurden spezifische Antikörper eingesetzt. Diese wurden an Cellulose-Beads gekoppelt Zunächst wurden $40 \mu \mathrm{l}$ der Bead-Suspension mit $2000 \mu 100$ mM Phosphatpuffer, $\mathrm{pH}$ 7,2 (PP) gewaschen und bei $7400 \mathrm{rpm}$ zentrifugiert. Nach der Resuspendierung in PP wurde die Mao B mit einer Proteinkonzentration von $2,5 \mathrm{mg} / \mathrm{ml}$ unter Schütteln bei $21^{\circ} \mathrm{C}$ für eine Stunde inkubiert. Schließlich wurden die Beads jeweils 4-mal abzentrifugiert und resuspendiert, um ungebundene Mao B zu entfernen.

\section{Bestimmung der Enzymaktivität}

Um die Detektion unterschiedlicher Mao-B Aktivitäten in biologischen Proben zu simulieren, wurden in den Experimenten verschiedene Volumina der Mao B-gekoppelten Cellulose-Beads (40 $\mu \mathrm{l}$ bis $160 \mu \mathrm{l}$ ) eingesetzt. Bei allen Ansätzen wurde das Volumen mit PP auf $1000 \mu$ laufgefüllt und in 4 Fraktionen zu $250 \mu \mathrm{l}$ aufgeteilt und bei 7400 rpm zentrifugiert. Nach Zugabe von $600 \mu \mathrm{l}$ mM Benzylamin in PP wurden die Ansätze für $0,10,20,30$ min bei $37^{\circ} \mathrm{C}$ unter Schütteln inkubiert. Nach der Inkubation wurden die Beads abzentrifugiert und $500 \mu \mathrm{l}$ des Überstandes mit 17,3 $\mu \mathrm{l} 3 \mathrm{M}$ $\mathrm{KCl}$ (in PP) hinzugegeben, um eine $\mathrm{KCl}-$ Konzentration von $100 \mathrm{mM}$ zu erhalten (wichtig für das Potential der $\mathrm{Ag} / \mathrm{AgCl}$ Referenz). Danach wurde die $\mathrm{H}_{2} \mathrm{O}_{2}$-Konzentration amperometrisch bestimmt (Abb. 1).

Zur Verifizierung der Ergebnisse aus den amperometrischen Messungen wurde die $\mathrm{H}_{2} \mathrm{O}_{2}$-Konzentration mittels eines optischen Assays (Monoamine Oxidase Activity Kit, Sigma Aldrich) bestimmt.

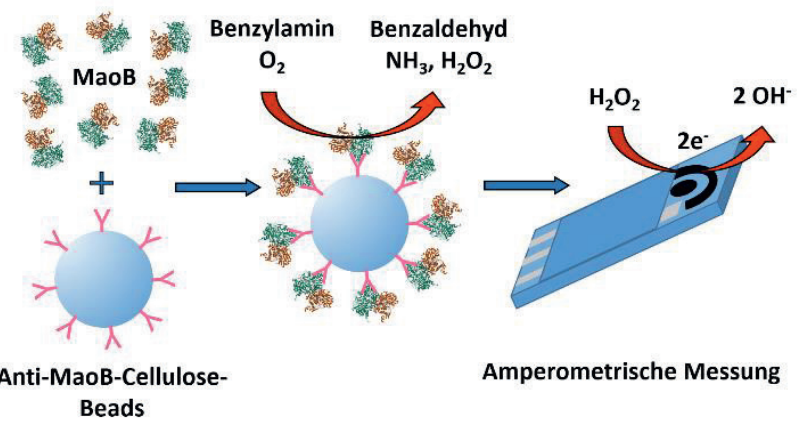

Abb. 1: Prinzip der Aktivitätsbestimmung der Mao B mittels amperometrischer Messung an Preußisch Blau-modifizierten Dickschicht-Elektroden von Rusens.

\section{Ergebnisse}

\section{Amperometrische Wasserstoffperoxid-Messungen}

Die amperometrische $\mathrm{H}_{2} \mathrm{O}_{2}$-Bestimmung wurde mit kommerziell erhältlichen Dickschichtelektroden durchgeführt, wobei eine Preußisch Blau-modifizierte Kohlenstoffelektrode mit einer Kohlenstoff-Gegenelektrode und einer Silber-Referenzelektrode kombiniert wird. Ein stabiles Referenzpotential ist dabei erst ab einer $\mathrm{KCl}$-Konzentration von 100 mM gewährleistet und wurde für alle amperometrischen Messungen verwendet. Für eine gute Reproduzierbarkeit war eine elektrochemische Vorbehandlung der Elektroden mit $100 \mu \mathrm{M} \mathrm{H}_{2} \mathrm{O}_{2}$ erforderlich.

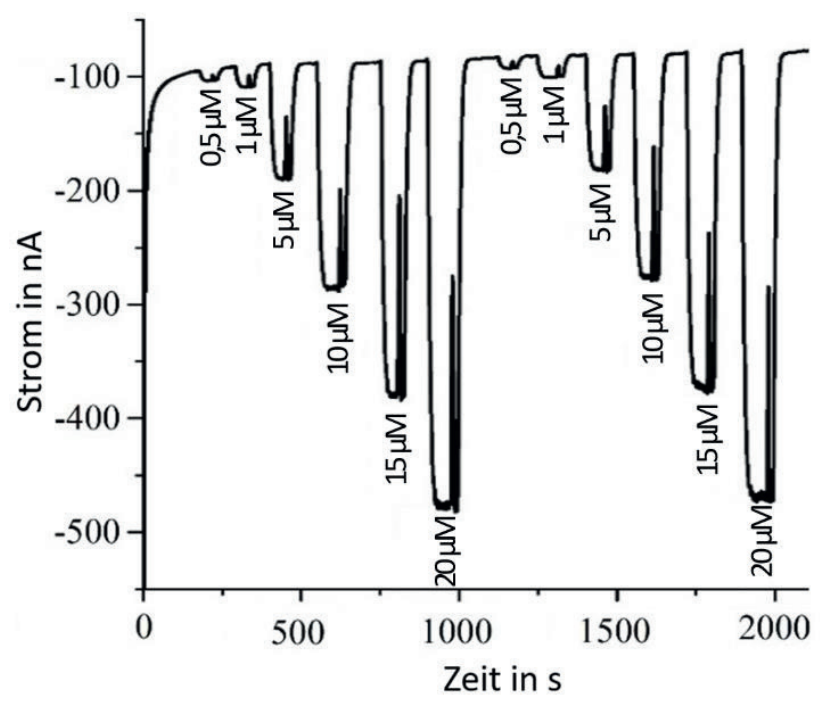

Abb. 2: Amperometrische Messung von $\mathrm{H}_{2} \mathrm{O}_{2}$ an vorhandelten Preußisch Blau-Dickschicht-Elektroden von Rusens in $100 \mathrm{mM}$ Phosphatpuffer ( $\mathrm{pH} \mathrm{7,2)} \mathrm{mit} 100 \mathrm{mM} \mathrm{KCl}$ bei einem Potential von $-100 \mathrm{mV}$ vs. $\mathrm{Ag} / \mathrm{AgCl}$ und einer Flussrate von $310 \mu \mathrm{min}^{-1}$.

Nach der Vorbehandlung konnte die Kalibrierung mit $\mathrm{H}_{2} \mathrm{O}_{2}$ erfolgen. Um die Signalstabilität zu zeigen, wurde die Kalibrierung dreimal in einem amperometrischen Experiment wiederholt (Abb. 2). Dabei ist festzustellen, dass das Signal mindestens 50 min sehr stabil ist und das amperometrische Messsignal linear von der $\mathrm{H}_{2} \mathrm{O}_{2}$-Konzentration abhängt 
(Abb. 3). Die Sensitivität der Preußisch Blau-modifizierten Elektroden sinkt von der ersten bis zur dritten Messung um nur 1 Prozent. Diese leichte Abnahme ist wahrscheinlich auf die Reduktion von Preußisch Blau zu Preußisch Weiß zurückzuführen, letzteres zeigt auf Elektroden eine geringere thermodynamische Stabilität (Karyakin, 2001).

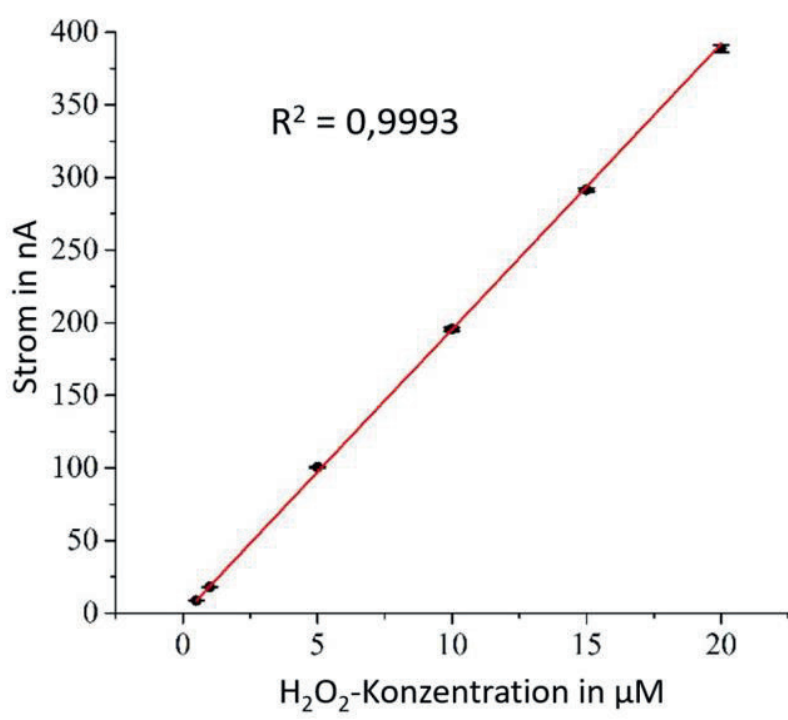

Abb. 3: Kalibrationskurve der Preußisch Blau-Dickschicht-Elektroden für $\mathrm{H}_{2} \mathrm{O}_{2}$.

Für die Bestimmung der Mao B-Aktivität mittels amperometrischer $\mathrm{H}_{2} \mathrm{O}_{2}$-Messung muss auch der Einfluss des Substrates Benzylamin und der Reaktionsprodukte Benzaldehyd und $\mathrm{NH}_{3}$ auf das Messsignal betrachtet werden. Während $\mu$-molare Konzentrationen von Benzaldehyd und $\mathrm{NH}_{3}$, wie sie beim Umsatz von Benzylamin durch die Mao $\mathrm{B}$ in den untersuchten Zeitspannen entstehen, keinen Einfluss auf die Signalstärke hatten, zeigte sich in Gegenwart von $1 \mathrm{mM}$ Benzylamin ein geringfügiger Anstieg um etwa $10 \mathrm{nA}$. Diese hohe Benzylaminkonzentration ist aber für das Messen der Enzymaktivität erforderlich. Um Fehler bei der $\mathrm{H}_{2} \mathrm{O}_{2}$-Bestimmung zu vermeiden, wurde den Lösungen zur $\mathrm{H}_{2} \mathrm{O}_{2}$-Kalibrierung $1 \mathrm{mM}$ Benzylamin hinzugegeben.

Nach dem Anpassen der Messlösungen konnten die Preußisch Blau-modifizierten Dickschicht-Elektroden mit hinreichender Spezifität für die Mao B-Aktivitätsmessungen mittels amperometrischer $\mathrm{H}_{2} \mathrm{O}_{2}$-Detektion eingesetzt werden.

\section{Amperometrische Mao B-Aktivitätsmessungen}

Spezifische Antikörper wurden dafür eingesetzt, das Enzym aus einer Lösung selektiv zu binden. Dabei wurde auch untersucht, ob die Antikörperbindung die enzymatische Aktivität beeinflusst. Für den Test können nur Antikörper eingesetzt werden, die weit genug vom aktiven Zentrum binden. Die Antikörper wurden für die Bindung der Mao-B an Cellulose-Beads gekoppelt. Dann wurde die gebundene Mao B für 10, 20 und 30 min in einer Lösung mit $1 \mathrm{mM}$
Benzylamin inkubiert und anschließend das gebildete Wasserstoffperoxid detektiert. Aus den amperometrischen Signalen wurden mittels der Kalibrierungsgeraden die betreffenden $\mathrm{H}_{2} \mathrm{O}_{2}$-Konzentrationen ermittelt.

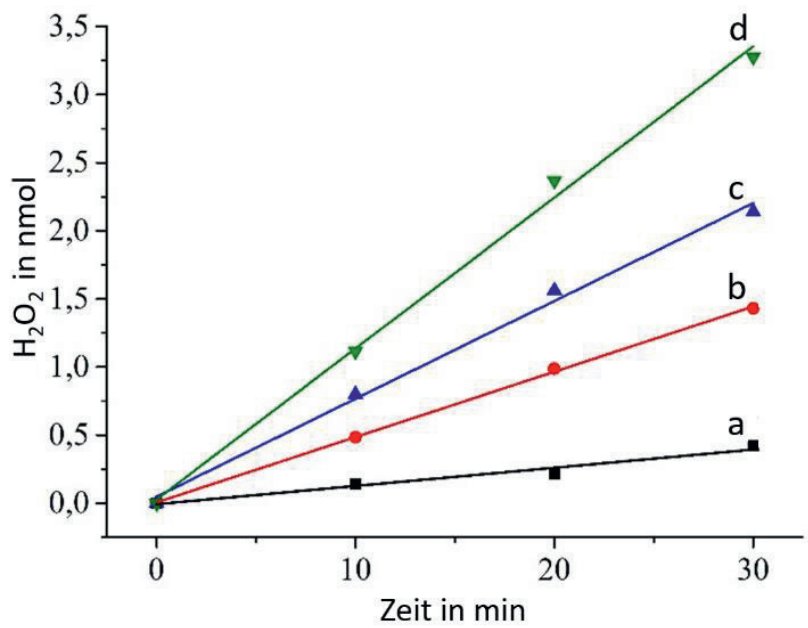

Abb. 4: Bestimmung der Aktivität der an mit Antikörpern modifizierten Cellulose-Beads gebundenen Mao B mittels amperometrischer Messung des beim enzymatischen Umsatz von Benzylamin entstehenden Wasserstoffperoxids. Die verschiedenen Geraden repräsentieren Messungen mit Proben, die (a) 1.6; (b) 2.3; (c) 3.2 or (d) $4.8 \%(v / v)$ Cellulose -Beads mit gebundener Mao B enthalten.

Wie in Abbildung 4 zu sehen, besteht ein linearer Zusammenhang von gemessener $\mathrm{H}_{2} \mathrm{O}_{2}$-Konzentration und Zeitdauer der enzymatischen Reaktion. Damit kann gezeigt werden, dass das hier vorgestellte System in der Lage ist, die Mao M mittels Antikörper-modifizierten Cellulose-Beads zu binden und deren Aktivität quantitativ zu erfassen.

Für diagnostische Anwendungen ist außerdem erforderlich unterschiedliche Aktivitäten des Enzyms mit dem Sensorsystem bestimmen zu können. Um diese zu untersuchen, wurden unterschiedliche Mengen an Cellulose Beads gebundener Mao B mit Benzylamin inkubiert und das entstehende $\mathrm{H}_{2} \mathrm{O}_{2}$ amperometrisch detektiert. Dabei zeigt sich, dass für alle Enzymmengen die $\mathrm{H}_{2} \mathrm{O}_{2}$-Konzentration linear mit der Zeit steigt. Außerdem korreliert die $\mathrm{H}_{2} \mathrm{O}_{2}$-Produktionsgeschwindigkeit mit der eingesetzten Enzymmenge. Ohne Benzylamin ist nahezu kein amperometrisches Signal zu beobachten und somit keine Enzymaktivität festzustellen. Mit der hier entwickelten Methode lassen sich Enzymaktivitäten von $1^{*} 10^{-5}$ bis $1,4^{*} 10^{-4} U$ nachweisen.

Die Ergebnisse aus der amperometrischen Aktivitätsbestimmung wurden schließlich mit denen des oben beschriebenen fluoreszenz-basierten kommerziellen Tests verglichen, bei dem das gebildete $\mathrm{H}_{2} \mathrm{O}_{2}$ mit Hilfe von Peroxidase einen Fluoreszenzfarbstoff oxidiert. Beim Einsatz von 4,8 \% (v/v) Cellulose-Beads mit gebundener Mao B werden mit dem optischen Test $7,2 * 10^{-5}$ und mit dem elektrochemischen Verfahren $8,6^{*} 10^{-5}$ Enzymaktivität detektiert. Damit sind die Ergebnisse beider Methoden gut vergleichbar. 


\section{Diskussion}

Ziel dieser Entwicklung war die elektrochemische Aktivitätsbestimmung der Mao B, einem der Enzyme, die eine Schlüsselrolle bei der Behandlung von Morbus Parkinson haben. Dabei ist die Mao B aus einer Lösung durch antiMao B-IgG an Cellulose-Beads selektiv gebunden worden. Dieser Bindungsschritt ist zusätzlich vorteilhaft für die Detektion, da alle anderen Bestandteile einer realen Probe (z.B. Blut) weggewaschen werden können und somit die Enzymmessung unter genau definierten Bedingungen abläuft.

Mit dem gewählten Enzymsubstrat Benzylamin entsteht durch die enzymatische Umsetzung Benzaldehyd, $\mathrm{NH}_{3}$ und $\mathrm{H}_{2} \mathrm{O}_{2}$. Letzteres wird durch die Verwendung von PreuBisch Blau modifizierten Dickschichtelektroden detektiert und somit die Mao B-Aktivität bestimmt. Ein Einfluss der Reaktionsprodukte Benzaldehyd und $\mathrm{NH}_{3}$ auf das Messsignal kann bei den akkumulierten $\mu$-molaren Konzentrationen ausgeschlossen werden. Eine Verfälschung der Messung durch 1 mM Benzylamin kann durch die Zugabe einer entsprechenden Menge zu den Kalibrierungslösungen vermieden werden.

Schließlich ist das entwickelte Verfahren zur Aktivitätsbestimmung eingesetzt worden. Zeitabhängige Messungen zeigen den linearen Anstieg der $\mathrm{H}_{2} \mathrm{O}_{2}$-Konzentration über einen Zeitraum von 30 min sowie eine klare Diskriminierung unterschiedlicher Enzymaktivitäten. Die Sensitivität des elektrochemischen Systems erlaubt dabei bereits nach 10 min eine Quantifizierung. Der Bereich der nachweisbaren Enzymaktivität liegt bei $1^{*} 10^{-5}$ bis $1,4^{*} 10^{-4} \mathrm{U}$. Bei einer Enzymaktivität von $0,01 \mathrm{U}$ bis $0,015 \mathrm{U}$ je $10^{10}$ Thrombozyten [12] und einer Thrombozytenzahl von 15 bis $45^{*} 10^{10}$ je Liter Blut [13], erreicht die hier entwickelte Methode die erforderliche Sensitivität für die Bestimmung der Mao B-Aktivität in Blutproben.

Es lässt sich also sagen, dass das entwickelte Verfahren zur Bestimmung der Enzymaktivität mittels amperometrischer Messung des enzymatisch gebildeten an Wasserstoffperoxid-sensitiven Dickschichtelektroden funktioniert und somit als Grundlage zur Evaluierung der Wirksamkeit der Medikation bei der Parkinson-Erkrankung dienen kann. Dies eröffnet Perspektiven für eine individualisierte Behandlung von Patienten.

\section{Literatur}

(1) PRINGSHEIM, T.; JETTE, N.; FROLKIS, A.; STEEVES, T. D. L. The Prevalence of Parkinson's Disease: A Systematic Review and Meta-Analysis. Mov. Disord. (2014), 29 (13), 1583-1590

(2) POEWE, W.; SEPPI, K.; TANNER, C. M.; HALLIDAY, G. M.; BRUNDIN, P.; VOLKMANN, J.; SCHRAG, A.-E.; LANG, A. E. Parkinson Disease. Nat. Rev. Dis. Primers (2017), 3, 17013

(3) DICKSON, D. W.; BRAAK, H.; DUDA, J. E.; DUYCKAERTS, C.; GASSER, T.; HALLIDAY, G. M.;
HARDY, J.; LEVERENZ, J. B.; DEL TREDICI, K.; WSZOLEK, Z. K.; LITVAN, I. Neuropathological Assessment of Parkinson's Disease: Refining the Diagnostic Criteria. Lancet Neurol. (2009), 8 (12), 1150-1157

(4) OERTEL, W.; SCHULZ, J. B. Current and Experimental Treatments of Parkinson Disease: A Guide for Neuroscientists. J. Neurochem. (2016), 139, 325-337

(5) BINDA, C.; HUBALEK, F.; LI, M.; EDMONDSON, D. E.; MATTEVI, A. Crystal Structure of Human Monoamine Oxidase B, a Drug Target Enzyme Monotopically Inserted into the Mitochondrial Outer Membrane. FEBS Lett. (2004), 564 (3), 225-228

(6) ADOLFSSON, R.; GOTTFRIES, C.-G.; ORELAND, L.; WIBERG, A.; WINBLAD, B. Increased Activity of Brain and Platelet Monoamine Oxidase in Dementia of Alzheimer Type. Life Sciences (1980), 27 (12), 1029-1034

(7) REYESPARADA, M.; SCORZA, M.; SILVEIRA, R.; DAJAS, F.; CASSELS, B. 4-Dimethylaminophenethylamine, a Sensitive, Specific, Electrochemically Detectable Monoamine Oxidase-B Substrate. Life Sci. (1994), 54 (25), 1955-1963.

(8) GOEBEL, G.; TALKE, A.; AHNERT, U.; LISDAT, F. Electrochemical Activity Determination of $\mathrm{Ca}$ techol-O-Methyl Transferase by Selective Dopamine Detection. ChemElectroChem (2019), 6 (17), 4533-4540

(10) KARYAKIN, A. A. Prussian Blue and Its Analogues: Electrochemistry and Analytical Applications. Electroanalysis (2001), 13 (10), 813819

(11) CHEN, W.; CAI, S.; REN, Q.-Q.; WEN, W.; ZHAO, Y.-D. Recent Advances in Electrochemical Sensing for Hydrogen Peroxide: A Review. Analyst (2012), 137 (1), 49-58

(12) ALM, P. O.; KLINTEBERG, B.; HUMBLE, K.; LEPPERT, J.; SORENSEN, S.; THORELL, L. H.; LIDBERG, L.; ORELAND, L. Psychopathy, Platelet MAO Activity and Criminality among Former Juvenile Delinquents. Acta Psychiatr. Scand. (1996), 94 (2), 105-111

(13) GILES, C. The Platelet Count and Mean Platelet Volume. Br. J. Haematol. (1981), 48 (1), 31-37

\section{Danksagung}

Unser Dank gilt dem Bundesministerium für Wirtschaft und Energie, welches dieses Forschungs- und Entwicklungsprojekt (16KN041836) im Rahmen des zentralen Innovationsprogramm Mittelstand gefördert hat. 